\title{
Hydrogenation of benzene and toluene over supported rhodium and rhodium-gold catalysts
}

\author{
Sapar Konuspayev ${ }^{1}$, Minavar Shaimardan ${ }^{2}$, Nurlan Annas ${ }^{1}$, T.S. Abildin ${ }^{1}$, Y.Y. Suleimenov \\ I \\ ${ }^{1}$ Al-Farabi Kazakh National University, Almaty, Kazakhstan \\ ${ }^{2}$ L.N. Gumilyov Eurasian National University, Nur-Sultan, Kazakhstan
}

\begin{abstract}
Rhodium and rhodium-gold catalysts supported on amorphous aluminosilicates (ASA), titanium dioxide (rutile, $\mathrm{TiO} 2$ ) was prepared in two different ways: absorption and colloidal method. The catalysts were characterized by an inductively coupled plasma optical emission spectrometer (ICP-OES), transmission electron microscopy (TEM) and Xray diffraction (XRD). The activity and selectivity of the prepared catalysts were tested by the hydrogenation of benzene and toluene. Hydrogenation was conducted at a pressure of $4 \mathrm{MPa}$ and a temperature $80{ }^{\circ} \mathrm{C}$. The bimetallic $\mathrm{Rh}-\mathrm{Au} / \mathrm{ASA}$ catalyst prepared by the absorption method showed higher activity and selectivity in benzene hydrogenation reaction, the same catalyst prepared by the colloidal method demonstrated lower selectivity.
\end{abstract}

\section{Introduction}

The content of aromatic compounds formed during the reforming of gasoline reaches $50 \%$, of which $10 \%$ is benzene. Incomplete combustion of benzene-containing gasoline inside the gasoline engine forms a carcinogenic compound called benzopyrene. Euro standards require emission reductions along the European regulatory pathway. According to Euro-4 and Euro-5 stage the benzene content in gasoline should not exceed 1\%. One effective way to remove small amounts of benzene is selectively hydrogenate benzene to cyclohexane. From this point of view, it is necessary to create catalysts that selectively hydrogenate benzene in presence of other aromatic compounds (toluene, xylene, cumene, etc.).

The most active metal from platinum group for the hydrogenation of aromatic compounds is rhodium supported on various carriers. [1-9]. The [1] authors studied the possibility of adsorption of an aromatic ring on the active site during the hydrogenation and found that Benzene preferentially adsorbs at the bridge $20^{\circ}$ adsorption sites of $\mathrm{Rh}$ (111). [2] The adsorption of rhodium on traditional carriers $\mathrm{SiO}_{2}, \mathrm{Al}_{2} \mathrm{O}_{3}$, and $\mathrm{TiO}_{2}$ have been investigated and found that aromatic molecules are weakly adsorbed on the carriers and are readily hydrogenated by spill-over hydrogen at room temperature. [3] The authors used various rhodium salts adsorbed onto silica or onto palladium supported on silica (Pd$\mathrm{SiO}_{2}$ ) and prepared bimetallic catalysts were examined for their catalytic arene hydrogenation activities. [4] Nanoparticles of rhodium embedded in polyvinylpyrrolidone. [5] Authors planted rhodium in nanocrystalline hydroxyapatite. [6] Rhodium was supported 
on $\mathrm{TiO}_{2}$ carriers in the form of anatase and rutile. Recently, we have prepared and characterized rhodium catalysts supported on various carbon carriers and evaluated there catalytic performance in hydrogenation reactions of benzene and toluene [7], as a result, rhodium catalysts supported on activated carbon with a surface area of more than $500 \mathrm{~m}^{2} / \mathrm{g}$ were found to be active and selective catalysts that allow selective hydrogenation of benzene in the presence of other aromatic compounds. The authors [8] prepared catalysis [TPPTS $=\mathrm{P}\left(\mathrm{C}_{6} \mathrm{H}_{4}-\mathrm{m}-\mathrm{SO}_{3} \mathrm{Na}\right)_{3}$ ] with a water-soluble $\mathrm{Rh} / \mathrm{TPPTS}$ system in an aqueous / organic two-phase system and achieved high catalytic activity in the hydrogenation of benzene to cyclohexane. The influence of several parameters of the system on the hydrogenation reaction of biphasic benzene was investigated. The highest catalytic activity in this reaction was observed at a low volumetric ratio of the water-gasoline phase, i.e., at 0.4 [9]. The researchers obtained rhodium (0) nanoparticles supported on ceria (RhNPs) by reduction of $\mathrm{Rh}^{3+}$ ions on the surface of ceria in aqueous medium. The $\mathrm{RhNPs} / \mathrm{CeO}_{2}$ catalyst exhibited high activity in benzene hydrogenation under mild conditions. The hydrogenation of benzene in solvent free condition was carried out at a circulation frequency of $495 \mathrm{~h}^{-1}$, a hydrogen gas pressure of $\sim 0.3 \mathrm{MPa}$, at a temperature of $25.0 \pm 0.1^{0}$ C. $\mathrm{RhNPS} / \mathrm{CeO}_{2}$ was more active than RhNPs supported on titanium, zirconium, and hafnium.

Herein, catalytic use of rhodium supported on amorphous aluminosilicate and titanium oxide, as well as bimetallic rhodium-gold in hydrogenation of benzene and toluene was reported.

\section{Experimental part}

\subsection{Materials}

Aqueous solutions of $\mathrm{RhCl}_{3}$ and $\mathrm{HAuCl}_{4}$ (Sigma Aldrich) were used as the starting compounds of $\mathrm{Rh}$ and $\mathrm{Au}$. Titanium dioxide (rutile, $\mathrm{TiO}_{2}$ ) and amorphous synthetic aluminosilicate (ASA) called "Siral" were used as carriers. In the colloidal method, PVA (Sigma Aldrich, $\mathrm{M}=10000$ ) and $\mathrm{NaBH}_{4}$ (Sigma Aldrich) were used as stabilizing and reducing reagents.

\subsection{Catalyst preparation}

Mono and bimetallic catalysts were prepared by using incipient wetness impregnation («imp») and colloidal («coll») method. The solutions were added dropwise to the supports, and then heated at $80^{\circ} \mathrm{C}$ for $3 \mathrm{~h}$, dried at $100^{\circ} \mathrm{C}$, and oxidized for $3 \mathrm{~h}$ at $250^{\circ} \mathrm{C}$ in stream of $\mathrm{H}_{2}$. During the preparation of bimetallic catalysts, $\mathrm{Au}$ and $\mathrm{Rh}$ were absorbed sequentially (first $\mathrm{Au}$, then $\mathrm{Rh}$ ). In the colloidal method, metal salts were oxidized with a strong reducing agent of sodium borohydride $\left(\mathrm{NaBH}_{4}\right)$ in their aqueous solutions to obtain finegrained particles. A stabilizing agent, polyvinyl alcohol (PVA), was added to an aqueous solution of a metal salt with a relative mass ratio $(\mathrm{mPVA} / \mathrm{m}(\mathrm{Au}+\mathrm{M})=1.2)$. Then an excess amount of $\mathrm{NaBH}_{4}$ was added in the case of solid mixing $\left(\mathrm{nNaBH}_{4} / \mathrm{n}(\mathrm{Au}+\mathrm{M})=5\right)$. After 1 hour, the remaining $\mathrm{NaBH}_{4}$ residue was added to the support after complete removal and acidified with the addition of 10-2M HC1 solution for sol-immobilization. Depending on the required experimental conditions, the $\mathrm{pH}$ was adjusted to 1.0 or 3.5. For the bimetallic catalyst in both different methods, the total weight of the metal was $3 \%$ (by weight) and 50:50 (by percentage). 


\subsection{Hydrogenation unit}

Benzene hydrogenation was carried out in a batch autoclave under isobaric-isothermal conditions. The reactor is equipped with a sampler that allowed samples to be withdrawn during the reaction, and a turbine stirrer made it possible to exclude outer diffusion factors. Temperature was maintained by an ultrathermostat. The autoclave of the unit is equipped with an ultra-thermostat layer, has a turbulent mixer to prevent the formation of waves, and a tubular neck, which allows the sample to be obtained during the reaction.

\subsection{Research methods}

The catalysts were characterized by inductively coupled plasma-optical emission spectroscopy (ICP-OES), transmission electron microscopy (TEM) and X-ray diffraction (XRD) methods.

\section{Results and discussion}

Two different methods have been used to absorb rhodium and gold from their salts. In the first method, rhodium and gold were impregnated sequentially (Au@Rh), and in the second, a mixture of gold and rhodium salts was impregnated simultaneously $(\mathrm{Au}+\mathrm{Rh})$. Table 1 shows the results of the ICP-OES analysis of the composition of the prepared catalysts.

Table 1. Chemical composition of $\mathrm{Au}-\mathrm{Rh} / \mathrm{TiO}_{2}$ (wt. \%) catalyst.

\begin{tabular}{|l|l|c|c|c|}
\hline Catalysts & Method of preparation & Au & Rh & Cl \\
\hline $\mathrm{Au} @ \mathrm{Rh}$ & Sequential impregnation & 2.1 & 0.73 & 0.31 \\
\hline $\mathrm{Au}+\mathrm{Rh}$ & Parallel impregnation & 2.33 & 0.73 & 0.3 \\
\hline $\mathrm{Au} @ \mathrm{Rh}$ & Colloidal sequent & 1.59 & 0.76 & 0.35 \\
\hline $\mathrm{Au}+\mathrm{Rh}$ & Colloidal parallel & 1.5 & 0.71 & 0.38 \\
\hline
\end{tabular}

It can be seen from this table that the amount of gold in the sequential impregnation method is higher than in the colloidal sequent method, where gold is 2.1 and 1.59; the rhodium content is vice versa, i.e., 0.73 and 0.76 . In the parallel impregnation method, the amount of gold and rhodium are also higher than in the colloidal method, i.e., 2.33 and 0.73 (gold 1.5 and 0.71). It is known that the amount of active metals impacts on activity of catalysts.

Fig. 1 shows the TEM images of the Au-Rh / ASA catalyst, based on these data (Fig.1-a, b), the size of the catalyst particles prepared by the colloidal method is $20 \mathrm{~nm}$, the size of the particles formed by the absorption method is $50 \mathrm{~nm}$. Fig.2-a, b show the TEM images of $\mathrm{Au}-\mathrm{Rh} / \mathrm{TiO}_{2}$ catalysts. It is notable that the sizes of metal particles in catalysts prepared by colloidal and absorption methods are 20 and $50 \mathrm{~nm}$ respectively. 


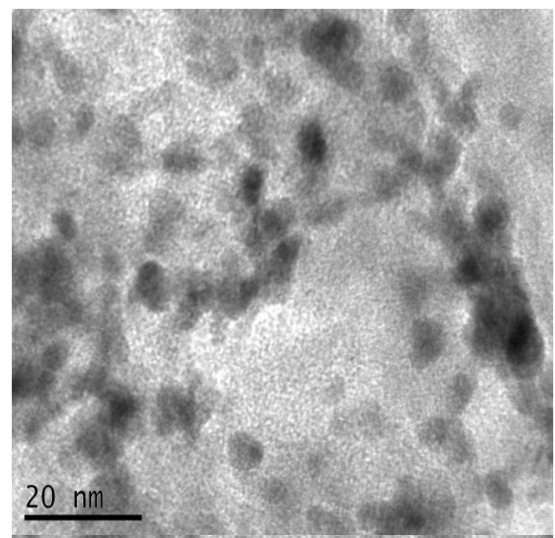

a. Colloidal method

Fig.1. TEM images of the Au-Rh/ASA catalyst

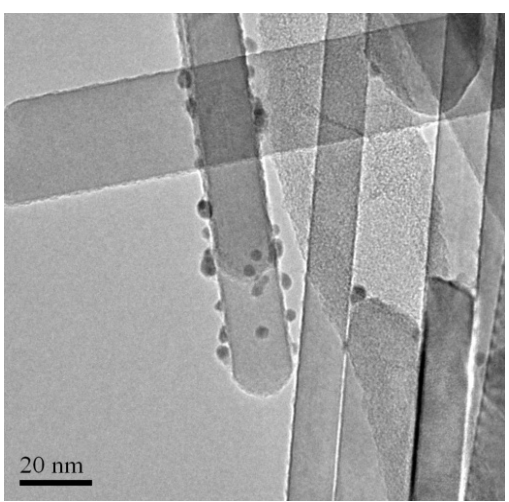

a. Colloidal

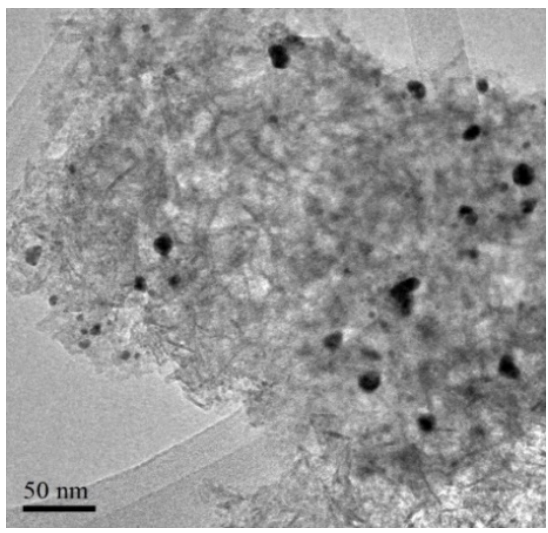

b. Impregnation method

Fig. 2. TEM images of the $\mathrm{Au}-\mathrm{Rh} / \mathrm{TiO}_{2}$ catalyst.

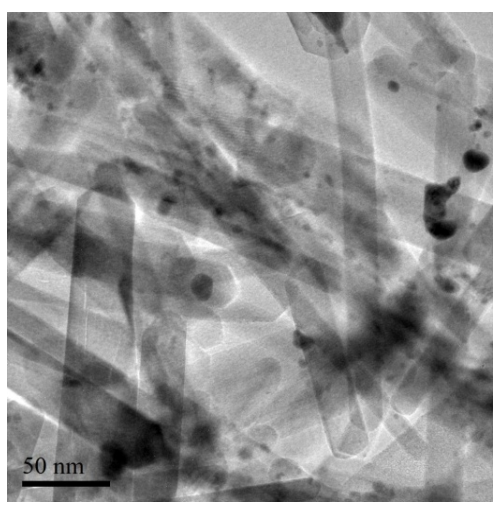

b. Impregnation 
XRD images of the Au-Rh/TiO 2 catalyst are shown in Fig.3. By analyzing XRD with high definition, the metallic phase of gold and rhodium can be seen separately, $\mathrm{TiO}_{2}$ is observed as rutile with a small amount of anatase impurity.

Although the XRD spectra are the same, there may be differences in composition or

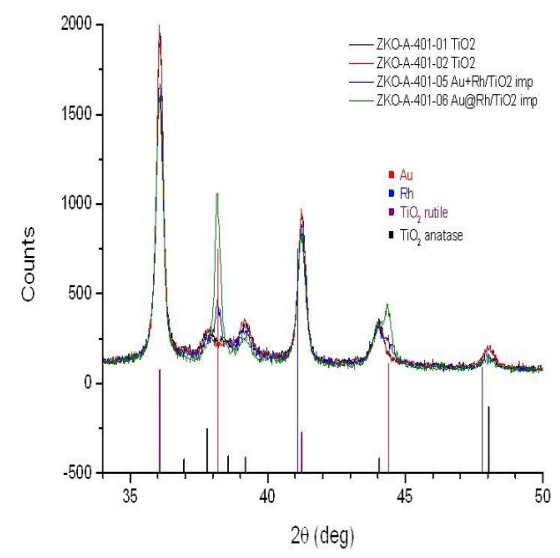

a. Impregnation method

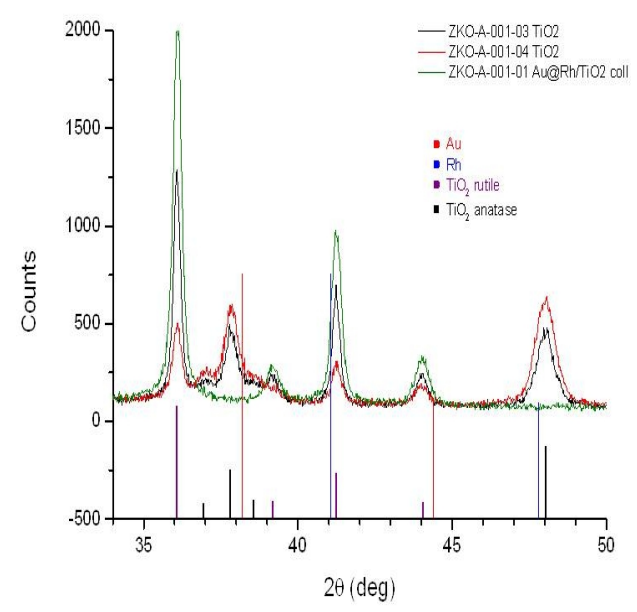

b. Colloidal method

Fig.3. XRD spectrum of the $\mathrm{Au}-\mathrm{Rh} / \mathrm{TiO}_{2}$ catalyst.

phases. In catalysts prepared by the impregnation method, phases of gold and rhodium can be observed; while in the colloidal method, these peaks deviate somewhat from pure metals, which can be explained by the presence of bimetallic particles of rhodium and gold as a solid solution and a intermetallic state in it.

To prepare a catalyst for the selective hydrogenation of benzene in the presence of other aromatic compounds, experiments were developed on the individual hydrogenation of benzene and toluene over prepared catalysts, following the principle that if a catalyst can selectively hydrogenate benzene in the presence of toluene, then it would be capable to selective hydrogenate benzene in the presence of other aromatic compounds.

Fig. 4 shows the curves of benzene hydrogenation using a rhodium catalyst (curve-1) supported on an amorphous aluminosilicate (ASA) carrier and a rhodium-gold catalyst (curve-2) supported on the same carrier. The catalysts are prepared by impregnation method. The shapes of the conversion curves are the same, the activity of individual rhodium is higher, i.e., it reaches $100 \%$ conversion in 220 minutes, and with the $\mathrm{Au}-\mathrm{Rh}$ catalyst it reaches $100 \%$ conversion in 240 minutes. It can be seen from this that with the same preparation method, the active metal of the catalyst is rhodium, addition of gold does not play an effective role. 


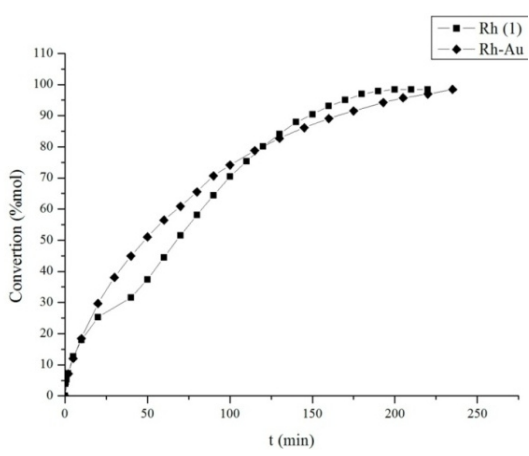

Fig.4. Hydrogenation of benzene at $4.0 \mathrm{MPa}, 80^{\circ} \mathrm{C}$ in ethanol over Rh/ASA (1) Au-Rh/ASA (2) catalysts prepared by the absorption method.

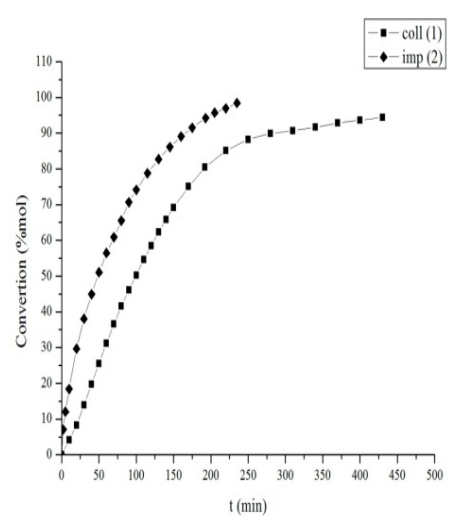

Fig.5. Hydrogenation of benzene at $4.0 \mathrm{MPa}, 80^{\circ} \mathrm{C}$ in ethanol over Au-Rh/ASA: 1-colloidal method; 2-absorption method.

The conversion curves of benzene hydrogenation with Au-Rh/ASA catalysts prepared by different methods are shown in Fig.5. It can be seen from the curves that they have the same shapes but exhibit different activity. While the complete conversion on the impregnated $\mathrm{Rh}-\mathrm{Au} / \mathrm{ASA}$ catalyst took 240 minutes, this colloidal catalyst took 430 minutes and only $95 \%$ conversion was achieved.

This can be explained by the state of the metal formed during the manufacture of the catalysts and the conditions for the formation of the active media of the catalysts. Firstly, when using the method of absorption, absorption of gold and rhodium ions into supports and its subsequent processing, they pass into the metallic state, and with the colloidal method, already formed metal particles (micelle nuclei) in a colloidal solution of rhodium and gold are planted into the supports. Secondly, when an aqueous solution of a metal salt is absorbed by amorphous silicon-aluminum, macro, micro and mesoporous deeper metal ions are formed. During the adsorption of the reduced metal from the colloidal solution, accumulations of metals are formed on the surface of the support. Thus, monodisperse metal clusters are obtained, but the mutual influence on the surfaces is limited due to the formation of coordinate bonds. The mutual influence arising from the absorption and pretreatment of the embedded catalysts leads to the formation of various phases, solid solutions, and spinel. During the oxidation of catalysts, all this affects the formation of a metal cluster and the state of charge of the active metal. As a result, it causes unequal activity and selectivity of the catalysts.

The hydrogenation conversion curves in $\mathrm{Rh}-\mathrm{Au} / \mathrm{ASA} \mathrm{Rh}-\mathrm{Au} / \mathrm{TiO}_{2}$ catalysts prepared by colloidal and absorption methods of benzene are shown in Fig.6 and 7. 


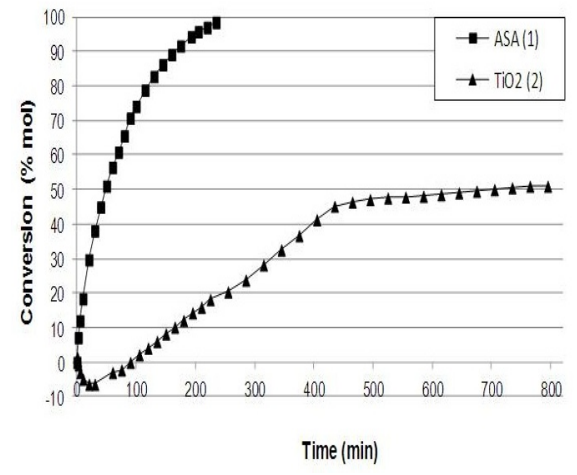

Fig. 6. Hydrogenation of benzene at $4 \mathrm{MPa}$ and $80{ }^{\circ} \mathrm{C}$ in ethanol over Au-Rh/ASA and Au-Rh / $\mathrm{TiO}_{2}$ catalysts prepared by the absorption method.

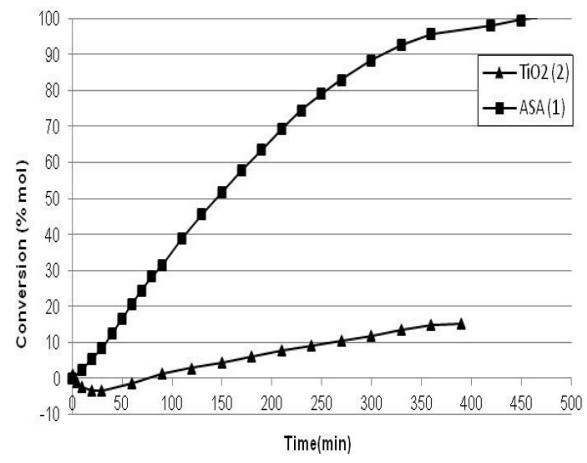

Fig. 7. Hydrogenation of benzene at $4 \mathrm{MPa}$ and $80{ }^{\circ} \mathrm{C}$ in ethanol over 1-Au-Rh/ASA and Au$\mathrm{Rh} / \mathrm{TiO}_{2}$ catalysts, prepared by colloidal method.

Fig. 6 shows that the conversion curve for similar catalysts prepared by the absorption method changes instantly (Fig. 6). 100\% conversion of benzene on Rh-Au / ASA is carried out within 240 minutes. On $\mathrm{Rh}-\mathrm{Au} / \mathrm{TiO}_{2}$ catalyst, the rate of the hydrogenation of benzene is slow, and the conversion curve is divided into three parts. In the first part, there is a reverse change in the volume of hydrogen, i.e., it gives the negative velocity. It can be assumed that the hydrogen started to be absorbed by the reaction medium. It is notable that, after 400 minutes of the reaction, the kinetic curve changes to a linear form and continues up to 800 minutes and the conversion of benzene was $50 \%$.

The catalysts prepared by the colloidal method (Fig. 7), the conversion of benzene over $\mathrm{Rh}-\mathrm{Au} / \mathrm{ASA}$ approached $95 \%$ within $425 \mathrm{~min}$, but barely reached $15 \%$ within $420 \mathrm{~min}$ on $\mathrm{Rh}-\mathrm{Au} / \mathrm{TiO} 2$ catalyst. It can be concluded that these catalysts are unsuitable for the quantitative hydrogenation of benzene.

Comparing the results of the figures (Fig.6,7), we can conclude that the most effective carrier for the active metal is amorphous silicon-aluminum (ASA) support for selective hydrogenation of benzene.

The conversion curves of toluene hydrogenation over the catalysts prepared by impregnation and colloidal methods are shown in Fig.8.

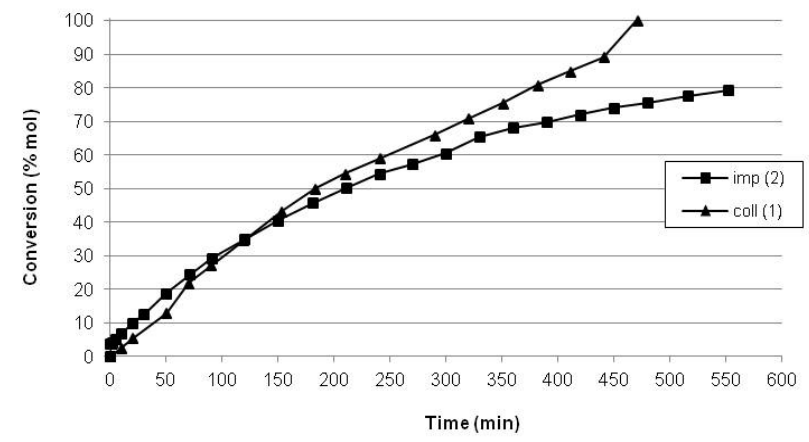

Fig. 8. Hydrogenation of toluene at $4.0 \mathrm{MPa}$ and $80^{\circ} \mathrm{C}$ in ethanol over Au-Rh/ASA catalysts: 1 prepared by the colloidal method; 2-prepared by the impregnation method. 
Comparing these curves with the curves of benzene hydrogenation (Fig.5), we note that the hydrogenation of toluene on a similar catalyst showed a completely different result. In fig. $8,100 \%$ conversion of toluene was achieved (curve 1) over the catalyst prepared by the colloidal method, while its conversation was only $77 \%$ on the catalyst prepared by the impregnation method in 550 minutes. (Fig. 5, to the complete conversion of benzene on the catalyst $\mathrm{Rh}-\mathrm{Au} / \mathrm{ASA}$ prepared by the impregnation method took 240 minutes), full conversion of toluene was not achieved.

According to the obtained results, we can conclude that Au-Rh/ASA prepared by the impregnation method is an effective catalyst for the selective hydrogenation of benzene in the presence of other aromatic compounds.

\section{Conclusion}

In this work, mono- and bimetallic catalysts $\mathrm{Rh}$ and $\mathrm{Au}-\mathrm{Rh}$ were prepared by two different methods, and their catalytic performance was tested in hydrogenation reactions. It is concluded that, the reaction activity of hydrogenation of benzene and toluene depends primarily on the nature of the active metal. The addition of the second component of gold could not increase the hydrogenation activity. The carrier also plays a role in the activity of the catalyst. It can be concluded that the activity of rhodium and rhodium-gold catalysts supported on aluminosilicate is slightly higher than that of similar catalysts supported on titanium oxide, which is explained by the specific surface area. The catalysts prepared by the impregnation method have a higher activity than those prepared by the colloidal method in the hydrogenation of benzene. ICP, TEM and XPS analysis showed that bimetallic rhodium-gold particles have a different structure than monometallic particles.

\section{References}

1. C. Morin, D. Simon, P. Sautet, J. Phys. Chem. B 108, 5653 (2004)

2. T. Ioannides and X. Verykios, J. Catal. 143, 175 (1993)

3. K.J. Stanger, Y. Tang, J. Andreegg, R.J. Angelici, J. Mol. Cat. A: Chemical 202, 147 (2003)

4. J-L. Pellegatta, C. Blandy, V. Colliere et al., J. Mol. Cat. A: Chemical 178, 55 (2002)

5. M. Zahmakiran, Y. Roman-Leshkov, Y. Zhang, Langmuir 28, 60 (2012)

6. V. Vishwanathan, M.S. Rajashekar, G. Sreekanth, S. Narayanan, J. Chem. Soc. Faraday Trans. 87, 3449 (1991)

7. S.R. Konuspaev, M. Shaimardan, D.R Nurbaeva., A.B Auezov, A.I. Boronin, Russ. J. Petrochem. 50, 48 (2010)

8. C. Vangelis, A. Bouriazos, S. Sotiriou, M. Samorski, B. Gutsche, G. Papadogianakis, J. Catal. 274, 21 (2010)

9. S. Akbayrak, J. Colloid Interface Sci. 530, 459 (2018) 